\title{
Fabrication of Bismuth Films by a Melt Spinning Method and the Influence of Annealing on Their Microstructure
}

\author{
G. N. Kozhemyakin ${ }^{a, b}, *$, S. Yu. Kovalev ${ }^{b, * *}$, and O. N. Soklakovac, *** \\ ${ }^{a}$ Shubnikov Institute of Crystallography, Federal Research Center Crystallography and Photonics, Russian Academy of Sciences, \\ Moscow, 119333 Russia \\ ${ }^{b}$ Lugansk National University, Lugansk, 91034 Ukraine \\ ${ }^{c}$ Belgorod State National Research University, Belgorod, 308034 Russia \\ *e-mail: genakozhemyakin@mail.ru \\ **e-mail:doomworld@mail.ru \\ ***e-mail: soklakova@bsu.edu.ru
}

Received November 20, 2018; revised February 18, 2019; accepted August 13, 2019

\begin{abstract}
The conditions of fabrication of bismuth films with thickness from 5 to $30 \mu \mathrm{m}$ by a melt spinning method were studied. The microstructure of the fabricated films in the cross section was analyzed using SEM. The Bi films with thickness more than $5 \mu \mathrm{m}$ consisted of crystallites with sizes up to $30 \mu \mathrm{m}$ and had a layered structure with crystal twins. The films with thickness of $\sim 5 \mu \mathrm{m}$ contained no crystallites, however, after annealing at $150-200^{\circ} \mathrm{C}$ for $1 \mathrm{~h}$, there appeared bismuth nanocrystals with sizes from 10 to $100 \mathrm{~nm}$. Annealing at higher temperatures promoted an increase in the size of nanocrystals and formation of crystallites of pyramidal shape with sizes up to $500 \mathrm{~nm}$.
\end{abstract}

Keywords: bismuth, melt spinning method, films, crystallites, annealing, nanocrystals

DOI: $10.1134 /$ S2075113320030259

\section{INTRODUCTION}

Bismuth as a semimetal is characterized by unique electrophysical properties owing to a specific energy band structure [1]. It is known that the Shubnikovde Haas effect was discovered for the first time in the studies of resistance of Bi single crystals in a magnetic field [2]. This was preceded by successful experiments on application of the Bridgman method for growing single crystals of bismuth and metals [3, 4].

As a consequence of a low crystallization point $\left(271^{\circ} \mathrm{C}\right)$, increase in a volume upon solidification, peculiarities of a crystalline structure, and anisotropy of thermal conductivity, the single crystals of $\mathrm{Bi}$ and its alloys with $\mathrm{Sb}$ are grown by directional crystallization: Bridgman method, zone melting, and Czochralski method [5-7]. However, only $\mathrm{Bi}-\mathrm{Sb}$ alloys have found practical application as highly efficient thermoelectric and magnetic thermoelectric materials. Despite the absence of practical application, pure $\mathrm{Bi}$ attracts interest from the point of view of analysis of its properties at low temperatures. Thus, for instance, in Bi films with $1-10 \mu \mathrm{m}$ thickness, a significant increase of magnetoresistance was detected at low temperatures, which can be used for fabrication of highly sensitive sensors of magnetic field and spintronic instruments [8,9]. Experimental data reveal that the Bi films with thickness of less than $50 \mathrm{~nm}$ are characterized by the properties of topological insulators [10].

In addition, $\mathrm{Bi}$ is an interesting material for analysis of transfer of charge carriers in low-dimensional structures. It is assumed that quantum effects in $\mathrm{Bi}$ can be detected at the nanostructure sizes of $\sim 50 \mathrm{~nm}$, which are larger than in other metals and semiconductors [11], resulting in intensive analysis of properties and development of fabrication methods of nanocrystalline $\mathrm{Bi}$ in the form of wires and ribbons [12, 13]. Nanocrystals can be obtained by annealing of amorphous metal alloys [14, 15]. Generally, amorphous metal alloys are fabricated by a melt spinning method; however, the data on application of this method to pure metals are limited [16, 17]. There are no published data on fabrication of bismuth semimetal by a melt spinning method.

The arm of this article is to study the conditions of fabrication of bismuth films by a melt spinning method and the influence of annealing on their microstructure.

\section{EXPERIMENTAL}

Bi films were fabricated by a melt spinning method on a specially developed machine (Fig. 1). Bi of $99.9999 \%$ purity was used as the source material. 


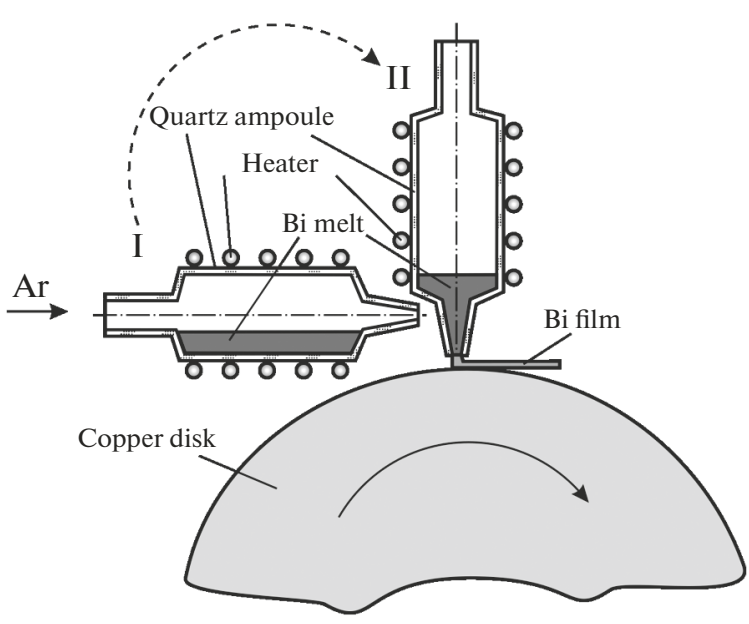

Fig. 1. Schematic illustration of bismuth spinning apparatus (I) horizontal position of the ampoule during Bi melting; (II) vertical position of the ampoule during melt spinning.

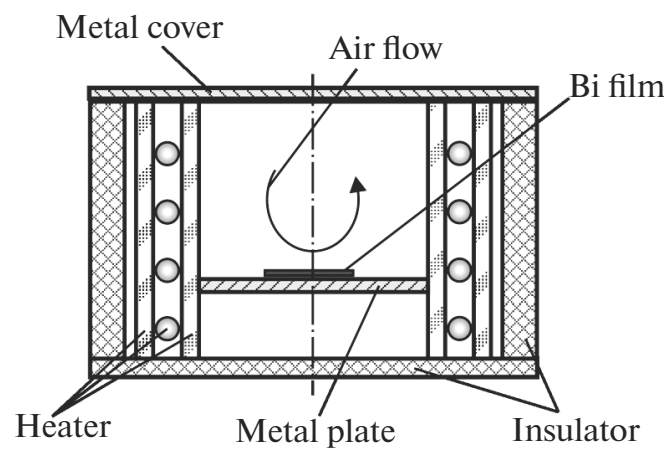

Fig. 2. Schematic illustration of furnace for annealing bismuth film.

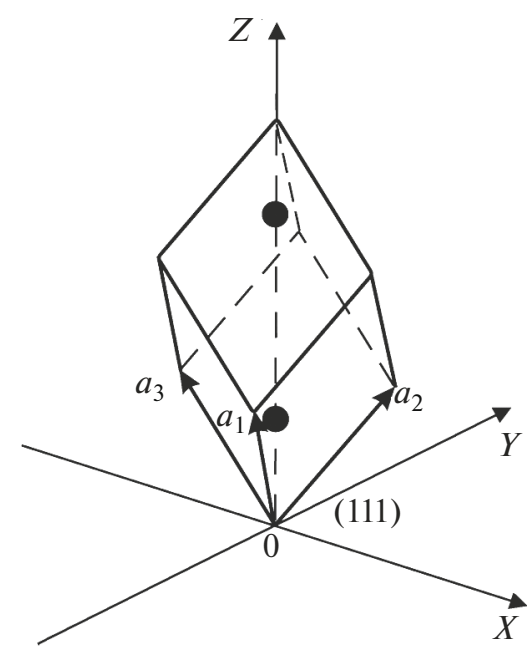

Fig. 3. Unit cell of bismuth crystal structure.

However, during transport and storage, the surface of the source Bi ingots was oxidized, and this oxide film after Bi melting prevented melt spinning. Thus, prior to application, the $\mathrm{Bi}$ was purified by droplet cleaning in vacuum, followed by directional crystallization for removal of the oxide film, increase in the resistivity ratio $\rho_{300 \mathrm{~K}} / \rho_{4.2 \mathrm{~K}}$ from 300 to 400 , and improvement of thermoelectric properties of $\mathrm{Bi}_{1-x} \mathrm{Sb}_{x}$ single crystals $[7,18]$. The polycrystalline Bi ingot fabricated in this way was broken into pieces with the mass of $\sim 7 \mathrm{~g}$, which were loaded into a quartz glass ampoule with $20 \mathrm{~mm}$ inner diameter and $115 \mathrm{~mm}$ length. A resistive nichrome heater was fixed on the outer surface of the ampoule; the heater was covered with ceramic insulating tube and maintained temperature of the melt. In order to prevent oxidation of the Bi melt, high purity $\mathrm{Ar}$ at a gauge pressure of $0.1 \mathrm{~atm}$ was passed through the ampoule. The Bi melt was held at $480-530^{\circ} \mathrm{C}$ for $15 \mathrm{~min}$ and then poured out onto a copper disk with $300 \mathrm{~mm}$ diameter at ambient temperature, which rotated at $1100 \mathrm{rpm}$. Owing to extra fast cooling of the melt, the Bi was fabricated in the form of films with the thickness of $5-30 \mu \mathrm{m}$, the width of $2-3 \mathrm{~mm}$, and the length of $15-35 \mathrm{~mm}$. The fabricated samples were annealed at 150,200 , and $220^{\circ} \mathrm{C}$ for $1 \mathrm{~h}$ in a special cylindrical furnace at a temperature gradient lower than $1 \mathrm{~K} / \mathrm{cm}$ in axial and radial directions (Fig. 2). The annealed and not annealed Bi film were brittle and were broken parallel to the plane (111) at minor mechanical loads [19]; hence, the morphology of the microstructure was analyzed on the cross section of $\mathrm{Bi}$ films obtained by breaking their in liquid nitrogen using a Quanta 200 3D scanning electron microscope.

\section{RESULTS AND DISCUSSION}

Bi semimetal is crystallized in a rhombohedral lattice and is assigned to the class of ditrigonal scalenohedron $D_{3 d}^{5}$; its unit cell contains two atoms (Fig. 3) [1]. The $Z$ axis corresponds to the direction of the $C_{33}$ trigonal axis, the $X$ axis corresponds to the $C_{11}$ binary axis, and the $Y$ axis corresponds to the $C_{22}$ bisectrix axis. The minimum distance between two nearest atoms of a unit cell corresponds to the direction $\langle 111\rangle$. The perfect cleavage plane (111) is perpendicular to the trigonal axis, and the rhombohedron faces are perfect cleavage planes. Bi crystals are characterized by a double layer structure (Fig. 4). The bond of the atoms in the layers is covalent with some portion of the metal bond. Metallic and van der Waals forces act between the layers, which decrease the strength of Bi single crystals prone to breakage parallel to the plane (111) $[7,19]$. The mentioned features of the Bi crystalline structure have an effect on the microstructure and strength of the films.

A characteristic feature of the microcrystalline structure of the fabricated $\mathrm{Bi}$ films is the existence of crystallites with size up to $30 \mu \mathrm{m}$ in the films with thickness more than $5 \mu \mathrm{m}$ (Fig. 5). In the films with thickness of $20-30 \mu \mathrm{m}$, these Bi crystallites had a layered structure and were formed in some regions in the form of crystal twins with the shape corresponding to 


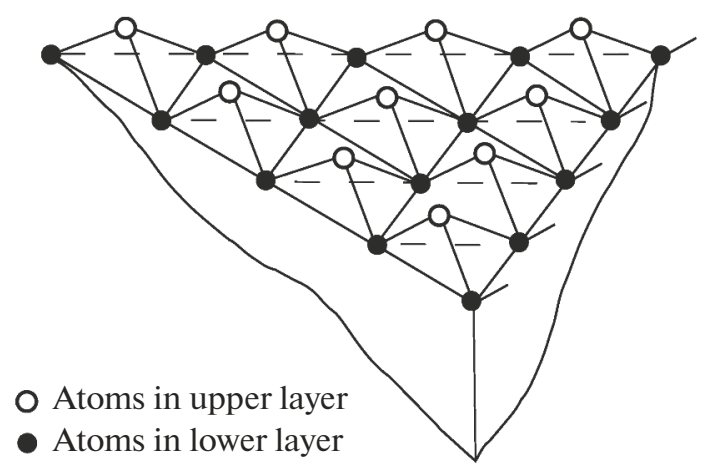

Fig. 4. Layered crystalline structure of bismuth.

a rhombohedral lattice (Fig. 5b). The Bi films with thickness about $5 \mu \mathrm{m}$ hardly contained any crystallites, which can characterize their structure as close to amorphous (Fig. 6). These Bi films did not contain cracks or a layered microstructure; however, it was possible to observe steps formed after their breaking in liquid nitrogen. One surface of all fabricated films, not contacting the copper disk upon cooling, was characterized by a wavy shape, which changed the film thickness (Figs. 5a, 6, 7).

Generally, nanocrystals in Bi films were formed only after annealing at $150^{\circ} \mathrm{C}$ and higher. Some nanocrystals with sizes from 10 to $100 \mathrm{~nm}$ were observed after annealing at $150^{\circ} \mathrm{C}$ both in the center and near film surface (Fig. 7). The increase in annealing temperature to 200 and $220^{\circ} \mathrm{C}$ resulted in an increase in the amount of nanocrystals with sizes of 50-100 nm and growth of crystallites with size up to $500 \mathrm{~nm}$. Under such growth conditions, most of the crystallites had the orientation at which the Bi plane (111) was perpendicular to the film surface. This is confirmed by the fact that the Bi crystallites formed on the cleavage surface after annealing had a pyramidal shape with the base of an equilateral triangle. Such shape of crystallites is close to the shape of the unit cell of the bismuth crystalline structure (Fig. 8). These crystallites were based on the plane (111), which was perpendicular to the film surface. Previously, it was established that Bi single crystals grow preferably with the orientation at which the plane (111) is parallel to the growth direction in accordance with a high axial temperature gradient [12]. It should be mentioned that $\mathrm{Bi}$ is characterized by anisotropy of thermal conductivity, the difference in its values reaching $70 \%$ depending on the orientation. In this case, the minimum thermal conductivity of Bi corresponds to the direction $\langle 111\rangle$, and the maximum thermal conductivity is parallel to the plane (111). Probably, such orientation of the grown crystallites is a consequence of the increase in the temperature gradient in the film directed perpendicular to its surface with the increase in annealing temperature as a consequence of more intense heat removal from the film surface due to the increase in convective mixing of air.

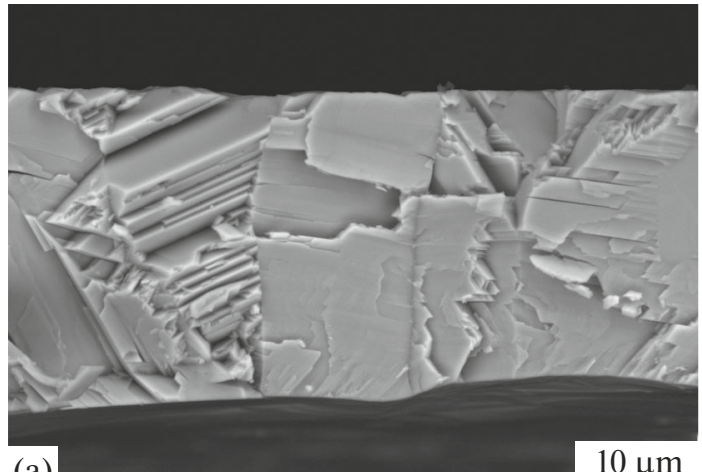

(a)

$10 \mu \mathrm{m}$

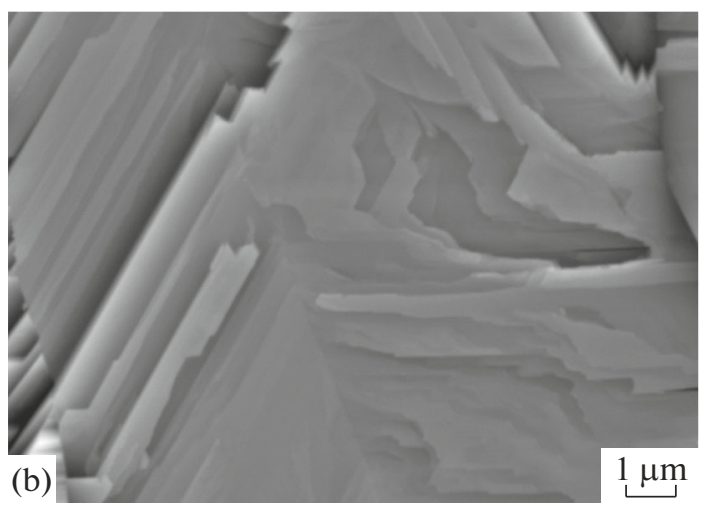

Fig. 5. Crystallites in bismuth films: (a) layered structure of crystallites; (b) the shape of crystal twins.

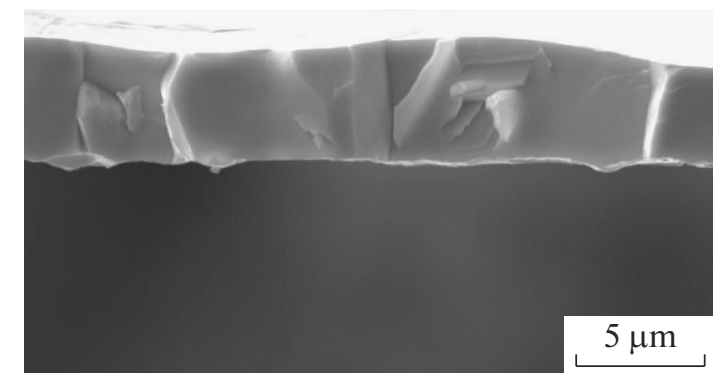

Fig. 6. Cross section of amorphous bismuth film before annealing.

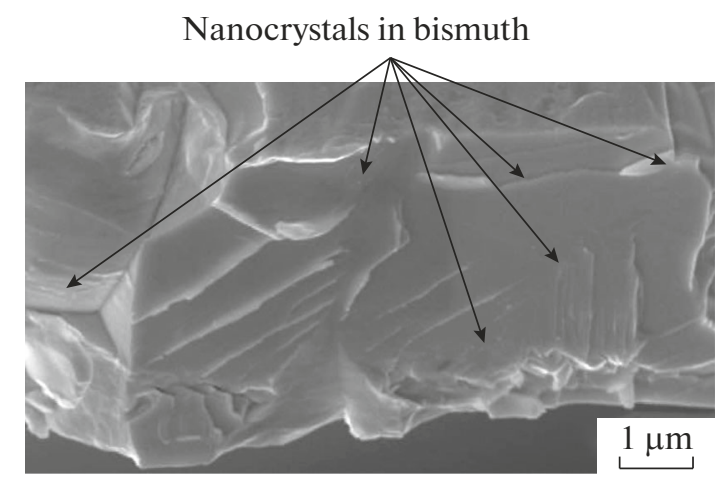

Fig. 7. Nanocrystals in bismuth film after annealing at $150^{\circ} \mathrm{C}$.

No. 32020 


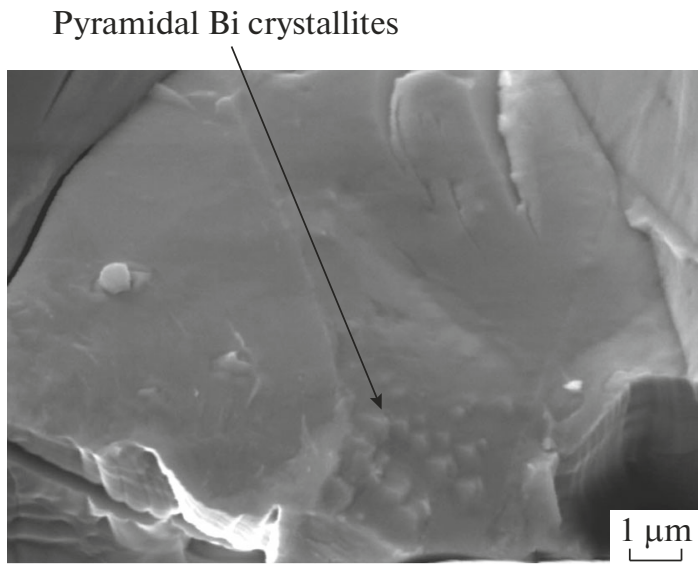

Fig. 8. Pyramidal crystallites of bismuth after annealing at $200^{\circ} \mathrm{C}$.

The detected features of the structure of Bi film obtained by a melt spinning method can be used for fabrication of bismuth films with nanocrystalline structure for application as topological insulators.

\section{CONCLUSIONS}

The apparatus and procedure for fabrication of $\mathrm{Bi}$ films with 5-30 $\mu \mathrm{m}$ thickness, $2-3 \mathrm{~mm}$ width, and $15-$ $35 \mathrm{~mm}$ length on a copper disk by a melt spinning method have been developed. High purity Ar was passed through the ampoule with Bi melt of $99.9999 \%$ purity to prevent $\mathrm{Bi}$ melt oxidization.

It has been established that the fabricated Bi films have a microcrystalline structure and consist of crystallites with sizes up to $30 \mu \mathrm{m}$. The films with thickness of more than $5 \mu \mathrm{m}$ have a layered microstructure, the shape of crystallites in them corresponds to the crystalline structure of $\mathrm{Bi}$, and they contain regions in the form of crystal twins. The Bi films with thickness of $\sim 5 \mu \mathrm{m}$ did not have a layered microstructure and they did not contain crystallites.

It has been shown that annealing of Bi films with the thickness of $\sim 5 \mu \mathrm{m}$ for $1 \mathrm{~h}$ at $150^{\circ} \mathrm{C}$ in air leads to formation of nanocrystals with the size of $10-100 \mathrm{~nm}$; however, upon annealing at 200 and $220^{\circ} \mathrm{C}$, in addition to nanocrystals, crystallites were generated with sizes up to $500 \mathrm{~nm}$ of pyramidal shape close to the shape of the unit cell of the Bi crystalline structure.

\section{REFERENCES}

1. Saikawa, K., Effective Hamiltonian describing the electronic states of bismuth-type crystals, J. Phys. Soc. Jpn., 1970, vol. 29, no. 3, pp. 562-569.

2. Schubnikov, L.V. and de Haas, W.J., A new phenomenon in the change of resistance in a magnetic field of single crystals of bismuth, Nature, 1930, vol. 126, no. 3179, p. 500 .
3. Obreimow, I.W. and Schubnikow, L.W., Eine Methode zur Herstellung einkristalliger Metalle, Z. Phys., 1924, vol. 25 , no. 1 , pp. $31-36$.

4. Kapitza, P., The study of the specific resistance of bismuth crystals and its change in strong magnetic fields and some allied problems, Proc. R. Soc. A, 1928, vol. 119, pp. 358-443.

5. Kuznetsov, V.D., Kristally i kristallizatsiya (Crystals and Crystallization), Moscow: Gostekhizdat, 1954.

6. Brown, D.M. and Heumaun, F.R., Growth of bismuth antimony single-crystal alloys, J. Appl. Phys., 1964, vol. 35, pp. 1947-1951.

7. Zemskov, V.S., Belaya, A.D., Beluy, Yu.S., and Kozhemyakin, G.N., Growth and investigation of thermoelectric properties of $\mathrm{Bi}-\mathrm{Sb}$ alloy single crystals, J. Cryst. Growth, 2000, vol. 212, pp. 161-166.

8. Yang, F.Y., Liu, R., Chien, C.L., and Searson, P.C., Large magnetoresistance and finite-size effects in electrodeposited single-crystal Bi thin films, Phys. Rev. Lett., 1999, vol. 82, pp. 3328-3331.

9. Semiconductors and Semimetals. Spintronics, Dietl, T., Awschalom, D.D., Kaminska, M., and Ohno, H., Eds., Amsterdam: Elsevier, 2008, vol. 82.

10. Demidov, E.V., Grabov, V.M., Komarov, V.A., Kablukova, N.S., and Krushelnitskii, A.N., Topological insulator state in thin bismuth films subjected to plane tensile strain, Phys. Solid State, 2018, vol. 60, no. 3, pp. 457-460.

11. Heremans, J., Thrush, C.M., Lin, Y.M., Cronin, S., Zhang, Z., Dresselhaus, M.S., and Mansfield, J.F., Bismuth nanowire arrays: synthesis and galvanomagnetic properties, Phys. Rev. B, 2000, vol. 61, pp. 2921-2930.

12. Meglei, I.D., Kantser, V., Dantu, M., Rusu, A., and Donu, S., High magnetoresistance in inhomogeneous bismuth microwires, Mold. J. Phys. Sci., 2008, vol. 7, no. 1 , pp. $61-63$.

13. Ning, W., Kong, F., Hi, C., Graf, D., Du, H., Han, Y., Yang, J., Yang, K., Tian, M., and Zhang, Y., Evidence of topological two-dimensional metallic surface states in thin bismuth nanoribbons, ACS Nano, 2014, vol. 8, no. 7 , art. ID 7506-12.

14. Noskova, N.I., Ponomareva, E.G., Glazer, A.A.Г., Lukshina, V.A., and Potapov, A.P., Effect of preliminary deformation and low temperature annealing on the size of $\mathrm{Fe}_{73.5} \mathrm{Cu}_{1} \mathrm{Nb}_{3} \mathrm{Si}_{13.5} \mathrm{~B}_{9}$ nanocrystals obtained by crystallization of an amorphous ribbon, Fiz. Met. Metalloved., 1993, vol. 76, no. 5, pp. 171-173.

15. Kozhemyakin, G.N., Shapovalov, V.A., Nikitenko, Yu.A., Ivanov, O.N., Kolesnikov, D.A., and Maradudina, O.N., Influence of annealing on nanocrystal formation in $\mathrm{Ni}$ amorphous alloy, Cryst. Rep., 2009, vol. 54, pp. 1242-1244.

16. Inoue, A., Preparation and novel properties of nanocrystalline and nanoquasicrystalline alloys, Nanostruct. Mater., 1995, vol. 6, pp. 53-64.

17. Kovneristyi, Yu.K., Ob”emno-amorfiziruyushchiesya metallicheskie splavy (Volume Amorphizing Metal Alloys), Moscow: Nauka, 1999.

18. Zemskov, V.S., Belaya, A.D., and Kozhemyakin, G.N., The effect of uncontrollable impurities on thermoelectric properties of Bi-9 at. \% Sb alloys, Izv. Akad. Nauk SSSR, Met., 1984, no. 5, pp. 194-195.

19. Ast, C.R. and Höchst, H., Fermi surface of Bi(111) measured by photoemission spectroscopy, Phys. Rev. Lett., 2001, vol. 87, no. 17, art. ID 177602.

Translated by I. Moshkin 